\title{
Characterization of Ge-doped optical fibres for MV radiotherapy dosimetry
}

\begin{abstract}
Ge-doped optical fibres offer promising thermoluminescence (TL) properties together with small physical size and modest cost. Their use as dosimeters for postal radiotherapy dose audits of megavoltage photon beams has been investigated. Key dosimetric characteristics including reproducibility, linearity, dose rate, temperature and angular dependence have been established. A methodology of measuring absorbed dose under reference conditions was developed. The Ge-doped optical fibres offer linearity between TL yield and dose, with a reproducibility of better than $5 \%$, following repeated measurements $(n=5)$ for doses from 5 cGy to $1000 \mathrm{cGy}$. The fibres also offer dose rate, angular and temperature independence, while an energy-dependent response of $7 \%$ was found over the energy range $6 \mathrm{MV}$ to $15 \mathrm{MV}$ (TPR20,10 of $0.660,0.723$ and 0.774 for 6,10 and $15 \mathrm{MV}$ respectively). The audit methodology has been developed with an expanded uncertainty of $4.22 \%$ at $95 \%$ confidence interval for the photon beams studied.
\end{abstract}

Keyword: Ge-doped optical fibres; Dosimetry intercomparison; Megavoltage photon 